Research Paper

\title{
JP3 enhances the toxicity of cisplatin on drug-resistant gastric cancer cells while reducing the damage to normal cells
}

\author{
Yi Zhang ${ }^{1,2}$, Junjie Chen ${ }^{1,2}$, Zhen Che ${ }^{1,2}$, Chuanjun Shu ${ }^{3}$, Dongyin Chen ${ }^{4}$, Kun Ding1,2, Aiping Li1,2, Jianwei \\ Zhou ${ }^{1,2}$ \\ 1. Department of Molecular Cell Biology \& Toxicology, Center for Global Health, School of Public Health, Nanjing Medical University, Nanjing 211166, China \\ 2. The Key Laboratory of Modern Toxicology, Ministry of Education, School of Public Health, Nanjing Medical University, Nanjing 211166, Jiangsu, China. \\ 3. Department of Bioinformatics, School of Biomedical Engineering and Informatics, Nanjing Medical University, Nanjing 211166, China. \\ 4. Department of Medicinal Chemistry, School of Pharmacy, Nanjing Medical University, Nanjing 211166, China.
}

$\triangle$ Corresponding author: Professor Jianwei Zhou, email: jwzhou@njmu.edu.cn. Department of Molecular Cell Biology \& Toxicology, Center for Global Health, School of Public Health, Nanjing Medical University, 101 Longmian Avenue, Jiangning District, Nanjing 211166, Jiangsu Province, China.

(0) The author(s). This is an open access article distributed under the terms of the Creative Commons Attribution License (https://creativecommons.org/licenses/by/4.0/). See http://ivyspring.com/terms for full terms and conditions.

Received: 2020.07.04; Accepted: 2021.01.03; Published: 2021.01.30

\begin{abstract}
Background: Cisplatin (DDP) is a highly effective chemotherapeutic agent to most solid tumors including gastric cancer (GC), however, its clinical value is limited due to severe toxic side effects and secondary drug resistance. JP3, a JWA protein based MMP2-targeted polypeptide, known to inhibit the growth of GC in vivo. However, the bidirectional effects of JP3 in DDP-resistant GC and normal cells have not been demonstrated. The present study aims to investigate the actions of JP3 on protecting normal cells from the toxicity of DDP while enhancing its anti-tumor effects on GC cells.

Methods: Routine laboratory experimental methods including CCK-8 assay, Western blotting, Hoechst staining, immunofluorescence (IF) and QRT-PCR were used in mechanism investigation; protein docking analysis and coimmunoprecipitation (Co-IP) were used for prediction and confirmation of interactions between JP3 and CK2. Mouse xenograft model was used for screening the treatment of JP3 plus DDP on GC growth.

Results: DDP showed similar toxicities to normal cells and DDP-resistant GC cells; JP3 competitively inhibited the binding of $X R C C 1$ to $C K 2$, reduced the DNA repair and anti-apoptosis capacity of DDP-resistant GC cells in combination with DDP treatment; meanwhile, JP3 protected normal cells from DDP-induced oxidative stress and DNA damage through ERK/Nrf2 signaling. JP3 combined with DDP showed similar bidirectional effects in vivo.

Conclusions: JP3 enhanced the inhibitory effects of DDP on tumor growth while reduced toxic side effects of DDP on normal cells. The results of this study provide a new insight for the treatment of drug-resistant GC.
\end{abstract}

Key words: Gastric cancer; cisplatin resistance; JP3 polypeptide; synergistic and detoxifying effect.

\section{Introduction}

Gastric cancer (GC) is the fifth commonly diagnosed cancer worldwide and third leading cause of cancer death [1]. In China, gastric cancer ranked first (13.6\%) among diagnosed cancers of the digestive tract, second $(13.5 \%)$ among diagnosed cancers in men and fifth (7.1\%) among women [2]. Currently, the major treatment for GC is chemotherapy plus surgical removal [3]. Cisplatin (DDP) is one of the first-line chemotherapeutic agents for GC. The limitations of DDP include drug resistance in cancer cells and toxicity to non-target tissues which hinder its clinical application [4]. The widely accepted mode of action for DDP is binding to cellular DNA and form DNA-DDP adduct, resulting in preventing DNA 
replication until the damage is repaired [5]. However, increased DNA repair in cancer cells is a main cause of DDP resistance [6]; and XRCC1 is a vital factor in single strand break repair (SSB) and base excision repair (BER) which causes the development of DDP resistance in various cancers [7]. Meanwhile, the toxicity of DDP including neurotoxicity, ototoxicity and gastrointestinal toxicity [8] have been attributed to various reasons which are, in turn, linked with reactive oxygen species (ROS) mediated oxidative stress [9]. Therefore, identification and development of novel agents that can sensitize GC cells to DDP and reduce damages to normal cells would have significant impact upon DDP-based chemotherapy.

JWA, also known as ARL6IP5, was initially cloned from human tracheal bronchial epithelial cells after treatment with all-trans retinoic acid [10]. Our previous data showed that in DDP-resistant GC cells, JWA down suppresses XRCC1 expression via CK2-p-XRCC1 signaling and enhances DDP-induced DNA damage and apoptosis [11]; while, in normal cells, JWA is involved in cellular responses to oxidative stresses and works as a DNA repair protein [12]. To find anticancer peptides that mimic JWA, we have recently designed and obtained an optimized polypeptide JP3. Our data showed that JP3 exerts therapeutic roles on GC through inhibition of angiogenesis via the TRIM25-SP1-MMP2 signaling [13]. Herein, we investigated the potential bidirectional roles and mechanisms of JP3 on DDP induced toxicity on drug-resistant GC cells and normal cells.

\section{Materials and methods}

\section{Cell cultures and reagents}

Human embryonic kidney cells HEK293T, human gastric epithelial cells GES-1, GC cell lines BGC823 and SGC7901 were purchased from the Type Culture Collection of the Chinese Academy of Sciences (Shanghai, China). We obtained the BGC323/DDP, SGC7901/DDP lines as described previously [14]. The BGC823, SGC7901, BGC323/ DDP, SGC7901/DDP and GES-1 cells were cultured in RPMI-1640 medium. The HEK293T cells were cultured in DMEM medium. All the cell lines were cultured with $100 \mathrm{U} / \mathrm{ml}$ penicillin and $100 \mu \mathrm{g} / \mathrm{ml}$ streptomycin and $10 \%$ fetal bovine serum. Cisplatin was obtained from Sigma-Aldrich (St. Louis, MO, USA). JP3 (peptide sequence: Ac-RMKKRYPTT(p) FVMVVGGGGHWGF-NH2; peptide purity: > 98.0\%) was synthesized by contracted service of Zhejiang Peptides Biotech Co., Ltd. (Hangzhou, Zhejiang, China).

\section{Cytotoxicity assay}

BGC823，SGC7901，BGC823/DDP， SGC7901/ DDP, GES-1 and HEK293T cells were plated at a density of 5000 cells per well in 96-well plates. On the next day, cells were treated with cisplatin at indicated concentrations. Cell Counting Kit-8 (CCK-8) was used to determine cell viability after $24 \mathrm{~h}$ treatments according to the manufacturer's instructions (Dojindo, Kumamoto, Japan). The cell variable curves were plotted and the $\mathrm{IC}_{50}$ values were evaluated by non-linear regression analysis with Graph Pad Prism software (La Jolla, CA, USA). The cell survival rates are expressed as the means \pm S.D. from at least three independent experiments.

\section{Apoptosis assay}

Apoptosis was determined by Hoechst 33342 staining (Beyotime Institute of Biotechnology, China). Cells were seeded in 12-well plates. After treatment with the indicated concentrations of cisplatin and/or JP3 for $24 \mathrm{~h}$, the cells were fixed with $4 \%$ paraformaldehyde and then stained with the Hoechst 33342 solutions for $15 \mathrm{~min}$ at $37^{\circ} \mathrm{C}$. Images were acquired under a Nikon fluorescence microscope. Uniformly stained nuclei were scored as healthy, viable cells. Condensed or fragmented nuclei were scored as apoptotic cells.

\section{Western blotting}

Whole cell lysates were prepared with RIPA lysis buffer containing a protease inhibitor cocktail (Roche, Manheim, Germany). Western blotting analysis was performed as previously described [15]. The following antibodies were used as follows: monoclonal anti-JWA (1:500, contract produced by AbMax, Beijing, China); polyclonal anti-PARP-1, anti- $\gamma-\mathrm{H} 2 \mathrm{AX}$, anti-XRCC1, anti-Bcl-2, anti-CK2a, anti-CAT, anti-Nrf2, anti-p-XRCC1 and p-ERK (1:1000-2000, Cell Signaling Technology, Danvers, MA, USA). Tubulin and GAPDH were used as loading controls.

\section{Measurement of GSH-Px, SOD and CAT}

Cells were incubated with cisplatin and/or JP3 for $24 \mathrm{~h}$. Glutathione peroxidase (GSH-Px), catalase (CAT), and superoxide dismutase (SOD) activity contents were estimated by the appropriate kits according to the manufacturer's protocols (Nanjing Jiancheng Bioengineering Institute, Nanjing, China). At least three biological replications were conducted for all experiments.

\section{Immunofluorescence microscopy}

After treatments, cells were fixed with $4 \%$ paraformaldehyde, followed by incubated with 
anti- $\gamma-\mathrm{H} 2 \mathrm{AX}$ (Cell Signaling Technology, Danvers, MA, USA) or 8-OHdG antibody (Santa Cruz, Dallas, TX, USA) monoclonal antibodies at a 1:200 dilution overnight at $4^{\circ} \mathrm{C}$. Then, the cells were incubated with FITC green-conjugated anti-rabbit IgG or Cy3 red-conjugated anti-mouse secondary antibody (1:400, Beyotime, Jiangsu, China) for $2 \mathrm{~h}$ at $37^{\circ} \mathrm{C}$. After washing with PBST, the nuclei were counterstained with DAPI (Beyotime, Jiangsu, China) for $15 \mathrm{~min}$. The confocal images of the cells were sequentially acquired with Zeiss AIM software on a ZeissQ7 LSM 700 confocal microscope system (Carl Zeiss Jena, Oberkochen, Germany).

\section{RNA extraction and reverse transcription-PCR}

Total RNA was extracted from the cells using TRIzol reagent (Gibco, Gaithersburg, MD, USA) and $500 \mathrm{ng}$ of RNA was used for a reverse transcription reaction with HiScript $Q$ RT SuperMix for qPCR (Vazyme, Jiangsu, China). The cDNA was amplified with the following primers: 5'- GGAGCGAGA TCCCTCCAAAAT -3' (forward) and 5'- GGCTGT TGTCATACTTCTCATGG -3' (reverse) for GAPDH; 5' - TGGAGCTGGTAACCCAGTAGG -3' (forward) and $5^{\prime}-$ CCTTTGCCTTGGAGTATTTGGTA $-3^{\prime}$ (reverse) for Catalase; $5^{\prime}$ - CAGTCGGTGTATGCCTTC TCG -3' (forward) and 5'- GAGGGACGCCACATT CTCG -3' (reverse) for GPx; 5' - TCAGCGACGGAA AGAGTATGA -3' (forward) and 5'-CCACTGGTT TCTGACTGGATGT -3' (reverse) for Nrf2; 5'GGTGGAATGGGGAAATCAAGAT $\quad-3^{\prime}$ (forward) and $5^{\prime}$ - TGATGATGTTGGGACCTCCTC $-3^{\prime}$ (reverse) for $\mathrm{CK} 2 \mathrm{a}$. GAPDH was used as the internal reference, and the results for each sample were normalized to GAPDH expression.

\section{Immunoprecipitation}

The cell extracts were centrifuged at $12,000 \times \mathrm{g}$ at $4{ }^{\circ} \mathrm{C}$ for $15 \mathrm{~min}$, and the supernatant was divided into two parts. One part was used for western blot, the remaining protein $(500 \mu \mathrm{g})$ had anti-XRCC1 antibody or IgG antibody ( $2 \mu \mathrm{g}$, Beyotime, Jiangsu, China) added and was cultured in $4{ }^{\circ} \mathrm{C}$ for $1 \mathrm{~h}$, and then incubated with Protein A/G agarose beads overnight. Afterwards, the beads were collected by centrifugation and washed four times at $1,000 \times \mathrm{g}$ for 5 min at $4{ }^{\circ} \mathrm{C}$ with precooling IP buffer. The immunoprecipitate was collected by centrifugation and resuspended in $1 \times$ SDS loading buffer and examined by western blot at last.

\section{Protein docking analysis}

I-TASSER (Iterative Threading Assembly Refinement) was designed for protein structure model by iterative threading assembly simulations. The molecular structures of CK2, JP3 and XRCC1 and the CK2a possible binding site for JP3 or XRCC1 were constructed by I-TASSER.

\section{BGC/DDP cells xenograft mice model}

Male BALB/c nude mice, 4-5 weeks old, weight 20-25 g were purchased from Model Animal Research Center of Nanjing University (Nanjing, China). The animal study proposal was approved by the Animal Care Committee of Nanjing Medical University, Nanjing, China. The mice were housed in a specific pathogen-free (SPF) environment (temperature $23 \pm 2^{\circ}$ C, $12: 12 \mathrm{~h}$ light/dark cycle and humidity $50 \pm 10 \%$ with free access to standard food and water.

After acclimation for one week, the mice were subcutaneously injected with BGC823/DDP GC cells $\left(5 \times 10^{6}\right)$ and when the average tumor volume reached $100-150 \mathrm{~mm}^{3}$ the mice were randomly divided into 4 groups including vehicle group, DDP group $(4 \mathrm{mg} / \mathrm{kg}$ every 3 days), JP3 group ( $25 \mathrm{mg} / \mathrm{kg}, \mathrm{Bid})$ and cisplatin+JP3 group. The mice body weight and tumor volume were measured every 3 days. Serum biochemical indicators were determined with an automatic biochemical analyzer according to the manufacturer's operating instructions. Pathological examinations of the major organs were performed by routine $H \& E$ staining.

\section{Statistics analysis}

All statistical analyses were performed with GraphPad Prism 6 software and/or SPSS 20.0. The data were expressed as the means \pm S.D. The differences among multiple groups were analyzed by one-way-ANOVA, and the differences between two groups were calculated using parametric unpaired Student's $\mathrm{t}$ test. $P<0.05$ was considered statistically significant.

\section{Results}

\section{DDP shows similar toxicities to normal cells and drug-resistant $\mathbf{G C}$ cells}

To understand the cytotoxicity of DDP on normal and cancer cells, we first evaluated the $\mathrm{IC}_{50}$ values of DDP by CCK-8 assay in six cell lines including normal cells (GES-1 and HEK 293T), GC cells (BGC823 and SGC7901), and DDP resistant GC cells (BGC823/DDP and SGC7901/DDP). As shown in Figure 1A, the survival rates of the cells were reduced in a dose-dependent manner after treatment with DDP at the indicated dose for $24 \mathrm{~h}$. The $\mathrm{IC}_{50}$ values of BGC823/DDP $(10.9 \mu \mathrm{g} / \mathrm{ml})$ and SGC7901/DDP $(8.49 \mu \mathrm{g} / \mathrm{ml})$ were significantly higher than their parental DDP-sensitive GC cells $(0.8 \mu \mathrm{g} / \mathrm{ml}$ for BGC823, $0.72 \mu \mathrm{g} / \mathrm{ml}$ for SGC7901) (Figure 1B); the 
resistance index (RI) of BGC823/DDP and SGC7901/DDP were 13.63 and 11.79, respectively. Importantly, although the $\mathrm{IC}_{50}$ value was higher in GES-1 $(12.3 \mu \mathrm{g} / \mathrm{ml})$ than that in DDP-resistant GC cells, it was obviously lower in HEK293T $(6.75 \mu \mathrm{g} / \mathrm{ml})$ than in DDP-resistant GC cells. To confirm the cytotoxicity of DDP on the cells, both DNA damage and apoptosis biomarkers were determined. The results showed that the expressions of both $\gamma$-H2AX and cleaved PARP1 in DDP-resistant GC cells and normal cells were dose-dependently increased after exposure of DDP at $0,1,3$ and $5 \mu \mathrm{g} / \mathrm{ml}$ for $24 \mathrm{~h}$ (Figure 1C-D). JWA expressions in these cells also showed a slight increase after DDP exposure. These results suggest that compared with cisplatin resistant GC cells, DDP showed similar toxicities to both normal cells. The toxicity of DDP to HEK293T cells was even greater than that to cisplatin resistant GC cells.

\section{JP3 plays a bidirectional role in DDP treated GC and normal cells}

JP3 is a functional phosphorylated fragment of JWA protein and linked with HWGF for targeting MMP2. To clarify whether JP3 exerted differential roles in DDP treated GC and normal cells, we completed cytotoxicity assays for the treatment of cisplatin in combination with JP3. Both BGC823/DDP (Figure 2A) and SGC7901/DDP (Figure 2B) cells were treated with either a fixed dose of DDP $5 \mu \mathrm{g} / \mathrm{ml}$ combined with a different dose of JP3 $(0,10,30,50$ $\mu \mathrm{g} / \mathrm{ml})$, or a fixed dose of JP3 $(50 \mu \mathrm{g} / \mathrm{ml})$ combined with a different dose of DDP $(1,3,5,9,12 \mu \mathrm{g} / \mathrm{ml})$ for $24 \mathrm{~h}$. The data showed that DDP induced a dose-dependent reducing in cell survival rates; however, the cell survival rates in JP3 combined with DDP treatment reduced more obvious than that in DDP exposure alone. These results suggest that JP3 dose-dependently enhanced the toxicity of DDP in both BGC823/DDP and SGC7901/DDP GC cells. The similar assay was conducted in GES-1 and HEK293T normal cells. As shown in Figure 2C, DDP treatment alone induced a dose-dependent toxicity in both cells; very interestingly, however, JP3 combined with DDP treatment significantly increased cell survival rates in both cells. The results suggested that JP3 partly protected normal cells from DDP induced damage.

To confirm the above data, we completed Hoechst assays. As shown in Figure 2D-E, compared to DDP treatment alone, JP3 enhanced pro-apoptotic toxicity of DDP in GC cells; in contrast, JP3 reduced pro-apoptotic toxicity of DDP in normal cells (Figure 2F-G). Further, the expressions of biomarkers indicated that DDP alone induced increasing protein levels of $\mathrm{y}-\mathrm{H} 2 \mathrm{AX}$ and cleaved PARP1; and these effects were further enhanced by JP3 in GC cells (Figure $2 \mathrm{H}$ ). However the expressions of $\mathrm{y}-\mathrm{H} 2 \mathrm{AX}$ and cleaved PARP1 were reduced by JP3 in normal cells (Figure 2I). In addition, JP3 also reduced the JWA expression levels in both GC and normal cells (Figure 2H-I). These results suggest that JP3 plays bidirectional effects in DDP treated GC and normal cells, respectively.
A

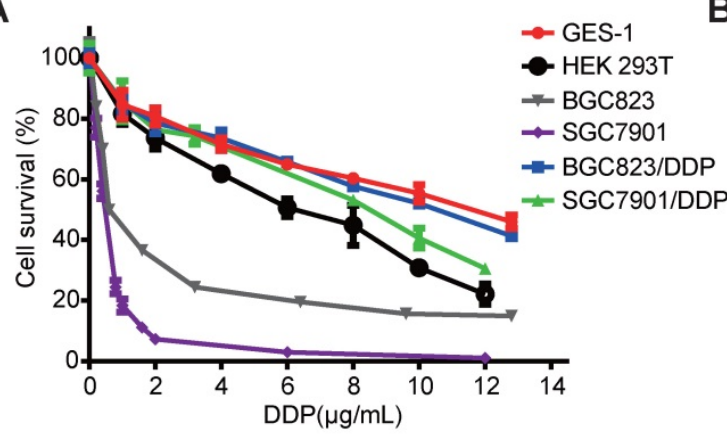

C

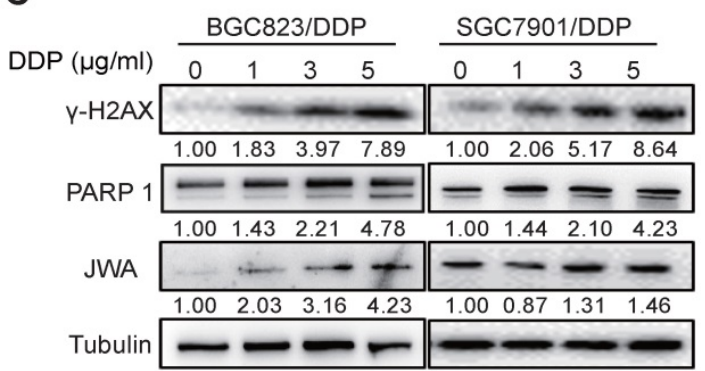

B

\begin{tabular}{cc}
\hline IC $_{50}$ & DDP $(\mu \mathrm{g} / \mathrm{ml})$ \\
\hline GES-1 & 12.3 \\
HEK 293T & 6.75 \\
BGC823/DDP & 10.9 \\
SGC7901/DDP & 8.49 \\
BGC823 & 0.8 \\
SGC7901 & 0.72 \\
\hline
\end{tabular}

D

\begin{tabular}{|c|c|c|c|c|c|c|c|c|}
\hline \multirow[b]{2}{*}{$\mathrm{DDP}(\mu \mathrm{g} / \mathrm{ml})$} & \multicolumn{4}{|c|}{ GES-1 } & \multicolumn{4}{|c|}{ HEK293T } \\
\hline & 0 & 1 & 3 & 5 & 0 & 1 & 3 & 5 \\
\hline$Y-H 2 A X$ & $5=$ & - & -1 & -1 & x- & - & $\sim$ & - \\
\hline & 1.00 & 1.49 & 2.56 & 5.37 & 1.00 & 2.32 & 5.76 & 9.65 \\
\hline PARP 1 & - & - & $=$ & $=$ & $=$ & 2 & 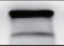 & $=$ \\
\hline & 1.00 & 1.40 & 3.59 & 6.04 & 1.00 & 1.41 & 1.83 & 4.13 \\
\hline JWA & $\cdots$ & $\cdots$ & - & 5 & $\rightarrow$ & 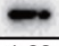 & - & - \\
\hline & 1.00 & 1.48 & 3.12 & 2.28 & 1.00 & 1.63 & 1.19 & 1.16 \\
\hline
\end{tabular}

Figure 1. DDP shows similar toxicities to normal cells and cisplatin-resistant GC cells. (A) IC 50 values of DDP in the indicated cell lines were determined. GES-1, HEK293T, BGC823, SGC7901, BGC823/DDP and SGC7901/DDP cells were treated with DDP (0-12 $\mu \mathrm{g} / \mathrm{ml})$ for $24 \mathrm{~h}$. (B) IC 50 values were calculated based on the results of cell viability measured by CCK-8. (C) BGC823/DPP, SGC7901/DDP, and (D) GES-1, HEK293T cells were treated with DDP at 0, 1, 3, $5 \mu \mathrm{g} / \mathrm{ml}$ for $24 \mathrm{~h}$. The protein levels of $\gamma-\mathrm{H} 2 \mathrm{AX}$, cleaved PARP-1 and JWA were detected by western blotting. Tubulin was used as the loading control. The intensities of protein bands were analyzed by densitometry after normalization to the corresponding tubulin levels. 

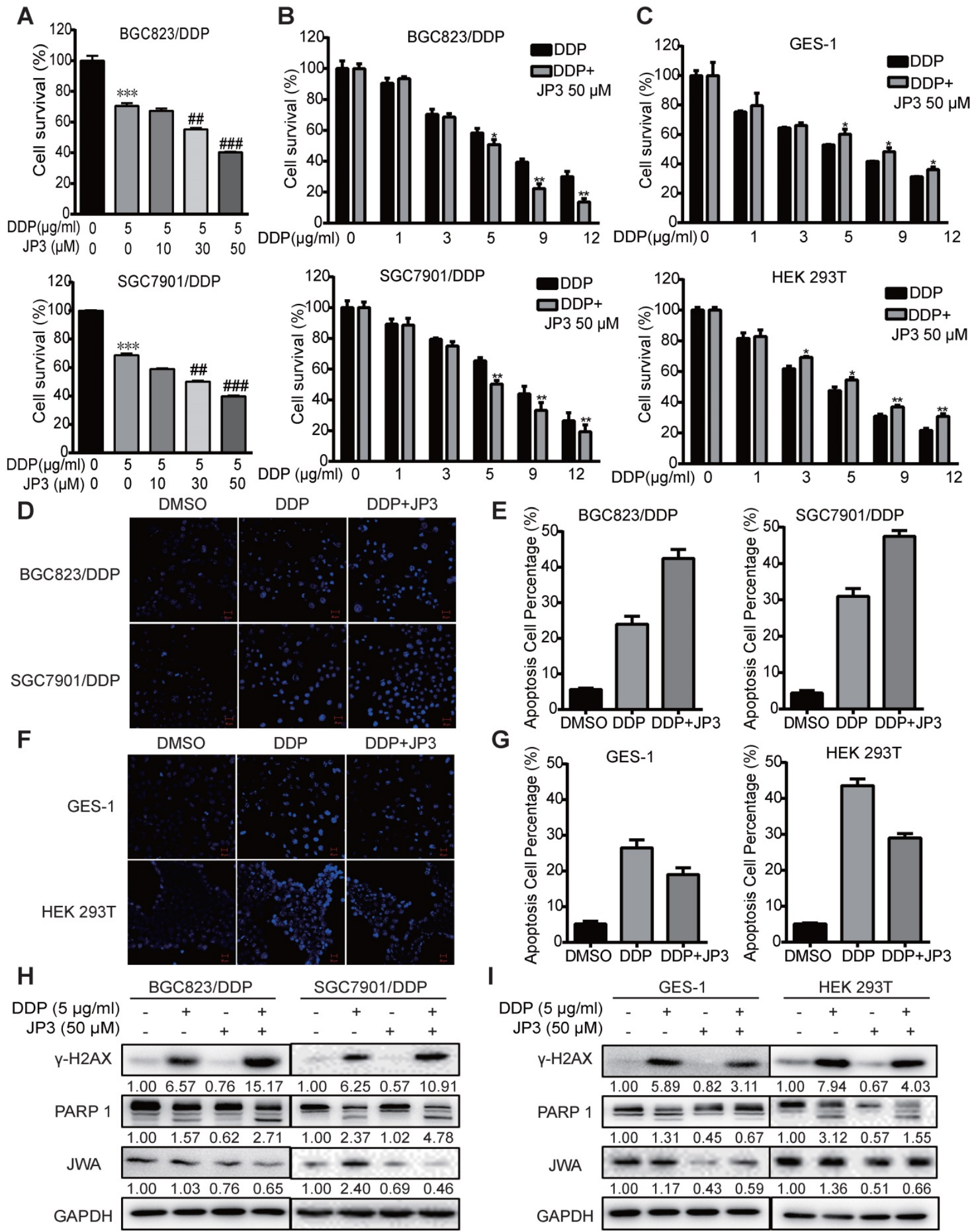

G

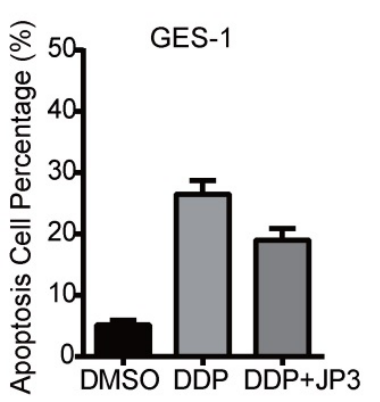

I

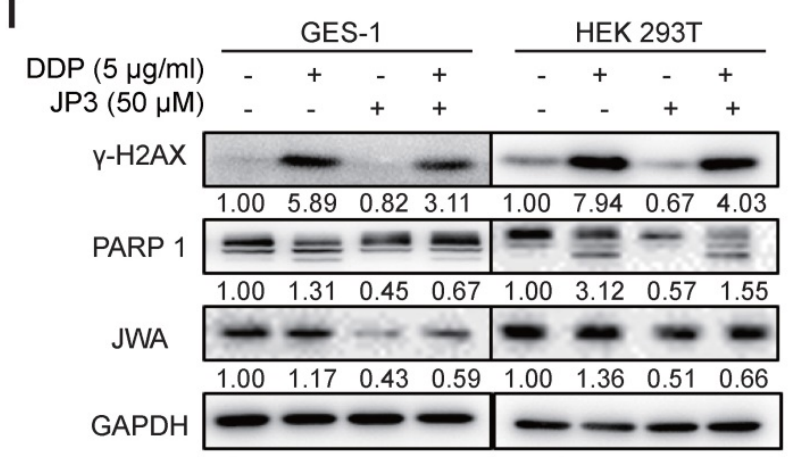

Figure 2. JP3 plays bidirectional roles in DDP treated GC and normal cells. (A) BGC823/DDP and SGC7901/DDP cells were treated with $5 \mu$ g/ml DDP and the indicated doses of JP3 for $24 \mathrm{~h}$; (B) BGC823/DPP, SGC7901/DDP, and (C) GES-1, HEK293T cells were treated with $50 \mu \mathrm{M}$ JP3 with or without different doses of DDP for 24 $\mathrm{h}$. The cell viability were measured by CCK-8 assay. BGC823/DDP, SGC7901/DDP (D, E) and GES-1, HEK293T cells (F, G) were treated with DMSO, $5 \mu \mathrm{gg} / \mathrm{ml}$ DDP or $50 \mu \mathrm{M}$ JP3 plus $5 \mu \mathrm{g} / \mathrm{ml}$ DDP for $24 \mathrm{~h}$, and the Hoechst staining images showed cell apoptosis. Scale bars $=100 \mu \mathrm{m}$. Quantitative data of apoptosis ratios of (D, F) were shown in (E, G), respectively. BGC823/DDP, SGC7901/DDP $(\mathrm{H})$ and GES-1, HEK293T cells (l) were treated with DMSO, $5 \mu \mathrm{g} / \mathrm{ml}$ DDP, $50 \mu \mathrm{MJP3}$ or DDP+JP3 for $24 \mathrm{~h}$. Protein levels of $\mathrm{Y}-\mathrm{H} 2 \mathrm{AX}$, cleaved PARP-1 and JWA were determined by western blot. GAPDH was used as the loading control. 
A

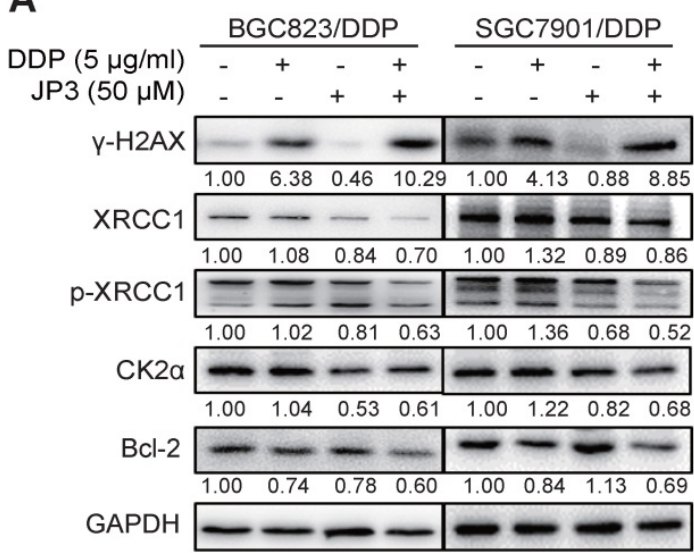

C

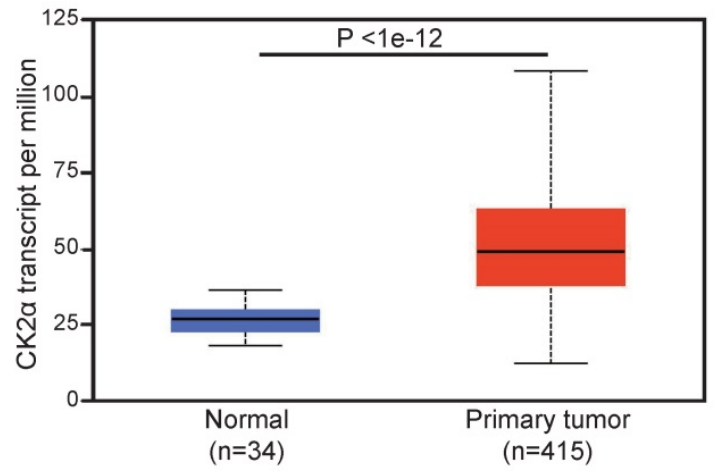

E

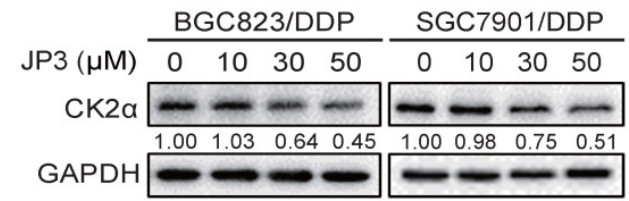

F

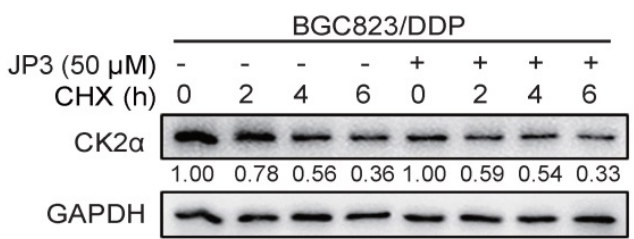

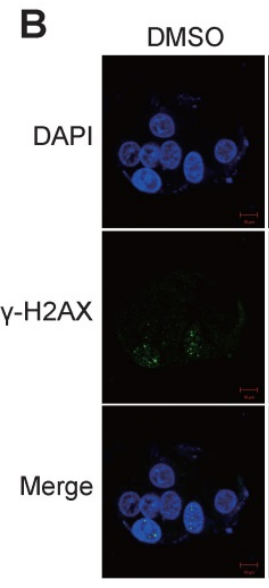

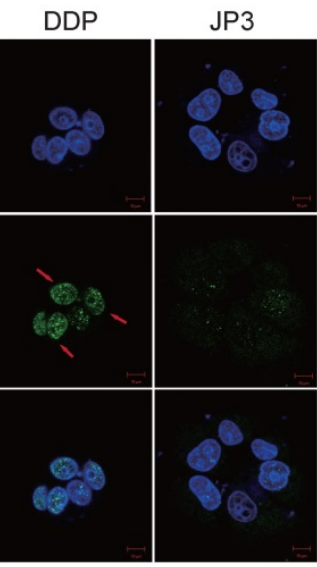

DDP $+J P 3$

D

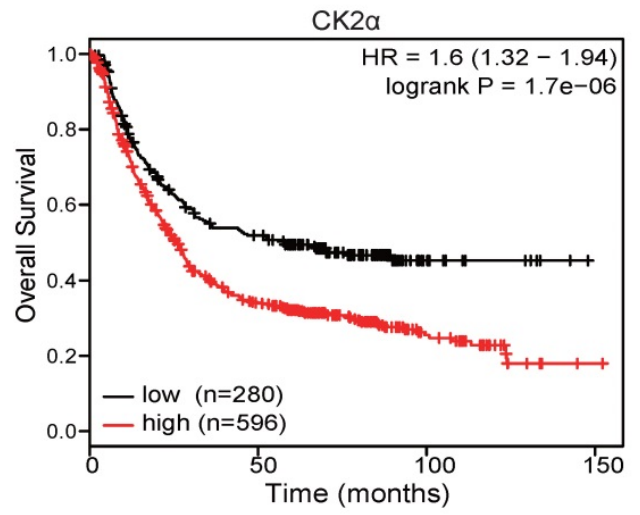

G
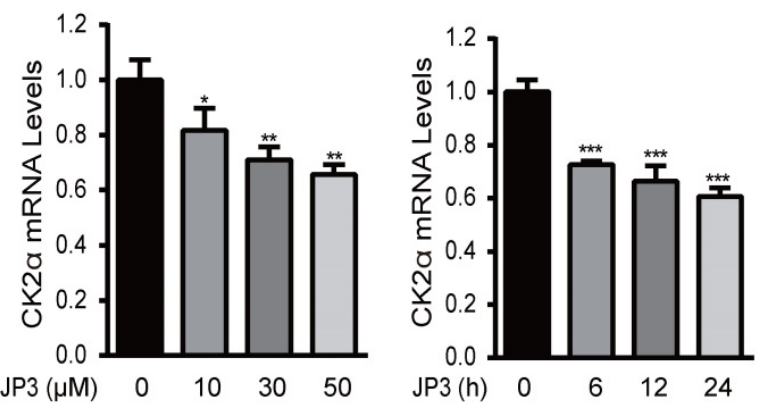

Figure 3. JP3 down-regulates CK2 expression and reduces XRCC1-mediated DNA repair in DDP-resistant gastric cancer cells. (A) BGC823/DDP and SGC7901/DDP cells were treated with DMSO, $5 \mu \mathrm{g} / \mathrm{ml}$ DDP, $50 \mu \mathrm{M} J \mathrm{P} 3$ or DDP+JP3 for $24 \mathrm{~h}$. Protein levels of $\mathrm{Y}-\mathrm{H} 2 \mathrm{AX}, \mathrm{XRCC1}$, P-XRCC1, CK2 $\alpha$ and Bcl-2 were determined by western blot. GAPDH was used as the loading control. (B) BGC823/DDP cells were treated with DMSO, $5 \mu \mathrm{g} / \mathrm{ml} \mathrm{DDP}, 50 \mu \mathrm{J}$ JP3 or DDP+JP3 for $24 \mathrm{~h}$ and immunofluorescence images showed $\mathrm{Y}-\mathrm{H} 2 \mathrm{AX}$ foci staining. Scale bars $=20 \mu \mathrm{m}$. (C) Expression of CK2 $\alpha$ mRNA in normal and primary GC tumor tissues were shown. (D) Kaplan-Meier curves depicting OS according to the expression patterns of CK2 $\alpha$ in GC cohort. $P$ values were calculated with the log-rank test. (E) BGC823/DDP and SGC7901/DDP cells were treated with $0,10,30,50 \mu \mathrm{M} J \mathrm{P} 3$ for $24 \mathrm{~h}$, respectively. Protein levels of CK2 $\alpha$ were determined by western blot. GAPDH was used as the loading control. (F) BGC823/DDP cells were treated with DMSO or $50 \mu \mathrm{MJP} 3$ for $24 \mathrm{~h}$ and then exposed to cycloheximide $(\mathrm{CHX})$ at $50 \mu g / \mathrm{ml}$ for the indicated times. CK2 $\alpha$ protein stability was determined by western blot. (G) BGC823/DDP cells were treated with $0,10,30,50 \mu \mathrm{M} J P 3$ for $24 \mathrm{~h}$ or $50 \mu \mathrm{M}$ JP3 for $0,6,12,24 \mathrm{~h}$; and then the mRNA levels of CK2 $\alpha$ were evaluated by qPCR assay. Error bars indicate the means \pm S.D. $* P<0.05, * * P<0.01, * * * P<0.001$.

\section{JP3 down-regulates CK2 expression and reduces $X R C C 1$-mediated DNA repair in DDP-resistant gastric cancer cells}

Our previous data showed that JWA down-regulates XRCC1 via ubiquitination of CK2, therefore reverses the resistance of GC cells [11]. Here, we determined if JP3 worked in GC cells through similar mechanism of JWA gene. As shown in Figure 3A, compared to DDP treatment alone, JP3 in combination with DDP significantly increased $\gamma$-H2AX level in DDP-resistant GC cells; on the contrary, expressions of XRCC1/p-XRCC1, CK2 $\alpha$ and $\mathrm{Bcl}-2$ were down-regulated in DDP-resistant GC cells. The immunofluorescence confocal assay also showed increased $\gamma$-H2AX foci in BGC823/DDP cells after treatment with JP3 plus DDP (Figure 3B), the quantified fluorescence intensity was shown in Figure S1A. The TCGA database showed that expression of CK2a was higher in 415 primary GC tumor samples 
than in 34 normal samples (Figure 3C, http:/ / ualcan. path.uab.edu/). We further constructed Kaplan-Meier survival curves (http://kmplot.com/analysis/) and found that the patients with high CK2a expression had poor OS compared with low CK2a ones $(P<0.01$; Figure 3D). Western blotting data showed the expression levels of CK2a were decreased by JP3 in dose dependent manner (Figure 3E). Moreover, CK2a was degraded more rapidly in JP3-treated BGC823/ DDP cells than in control cells (Figure 3F). Treatment of BGC823/DDP cells with MG132 increased the expression of CK2a; however, MG132 did not show effects on CK2 $\alpha$ by JP3 treatment (Figure S1C). To determine how JP3 reduced CK2 $\alpha$ expression, we firstly completed RT-PCR assays. Data showed that JP3 dose/time-dependently down-regulated CK2a expression in BGC823/DDP cells (Figure 3G).

\section{JP3 competitively inhibits interactions between CK2 $\alpha$ and XRCC1 in DDP-resistant GC cells}

Since the phosphorylation of XRCC1 by CK2 is required for its stability and efficient DNA repair in SSB [16]. To determine whether JP3 inhibited interactions between $\mathrm{CK} 2 \mathrm{a}$ and XRCC1, we completed computational molecular docking analysis. As a result, the interaction between CK2 $\mathrm{a}$ and JP3 was identified and the binding sites of the both were overlapped with those between XRCC1 and CK2a (Figure 4A-B). Immunofluorescence confocal images further confirmed that the co-localization between JP3 and CK2a was existed in both BGC823/DDP and SGC7901/DDP cells (Figure 4C). These results were also supported by co-IP assay in Figure 4D, Figure S1D, data showed the interaction between XRCC1 and CK2 was weakened in the cells treated by JP3 in combination with DDP compared to the cells treated by DDP alone. Suggesting that JP3 down-regulates expressions of CK2 $\alpha /$ XRCC1 through several mechanisms including at least transcriptional and competitive inhibitions.

\section{JP3 attenuates DDP-induced DNA damage in normal cells by reducing ROS production}

DDP is known to generate cytotoxicity and side effects in patients by oxidative stress and direct DNA damage related events [17]. Our previous data showed JWA as a DNA repair protein involves in regulation of oxidative stress and cell response process [18]. To determine if the protection roles of JP3 on normal cells from DDP toxicity was through its anti-oxidant mechanism, we conducted related assays. As shown in Figure 5A, JP3 reduced 8-OHdG levels by DDP in GES-1 cells compared with DDP treatment alone. Reactive oxygen species (ROS) also decreased by the treatment of JP3 plus DDP in GES-1 and HEK 293T cells compared with that by DDP alone (Figure 5B); in addition, the numbers of DNA damage foci and DCFH-DA positive cell were also reduced in JP3 plus DDP cells compared to DDP treatment alone. (Figure S2A-B).
A

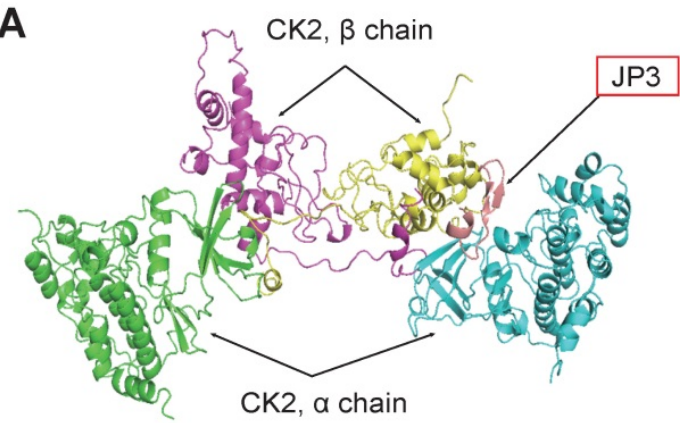

B

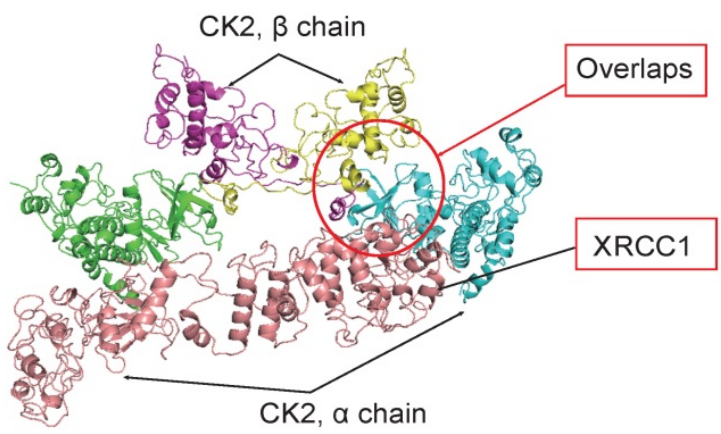

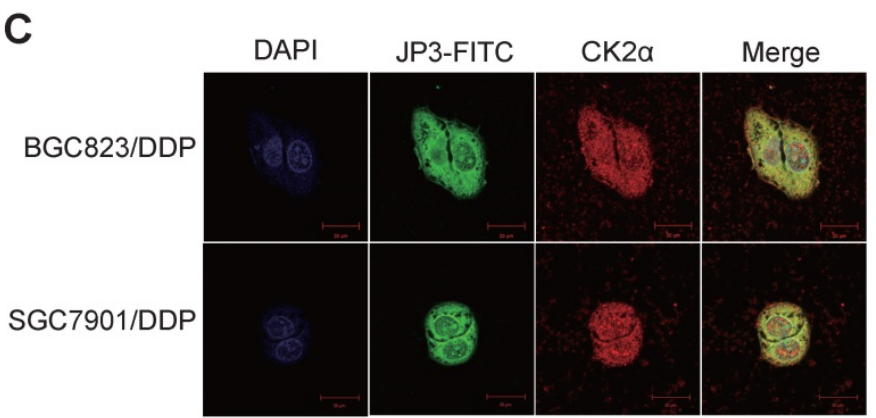

D BGC823/DDP

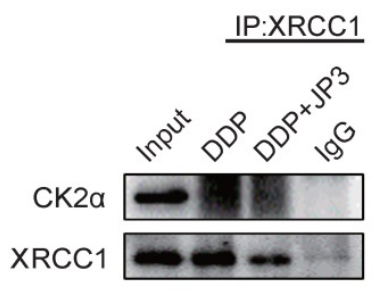

Figure 4. JP3 competitively inhibits interactions between CK2 $\alpha$ and XRCC1 in DDP-resistant GC cells. (A, B) Protein-protein interaction molecular dockings of CK2 $\alpha$ and JP3, CK2 $\alpha$ and XRCC1 were shown. (C) BGC823/DDP and SGC7901/DDP cells were treated with JP3-FITC for 6 h, and the representative immunofluorescence images showed the co-localizations among JP3-FITC (green), CK2 $\alpha$ (red) and DAPI (blue). Scale bars $=20 \mu \mathrm{m}$. (D) BGC823/DDP cells were pretreated with DDP or DDP+JP3 for $24 \mathrm{~h}$, and the endogenous protein-protein interaction between CK2 $\alpha$ and XRCCl was determined by co-IP with XRCCl antibodies followed by western blot confirmation. 


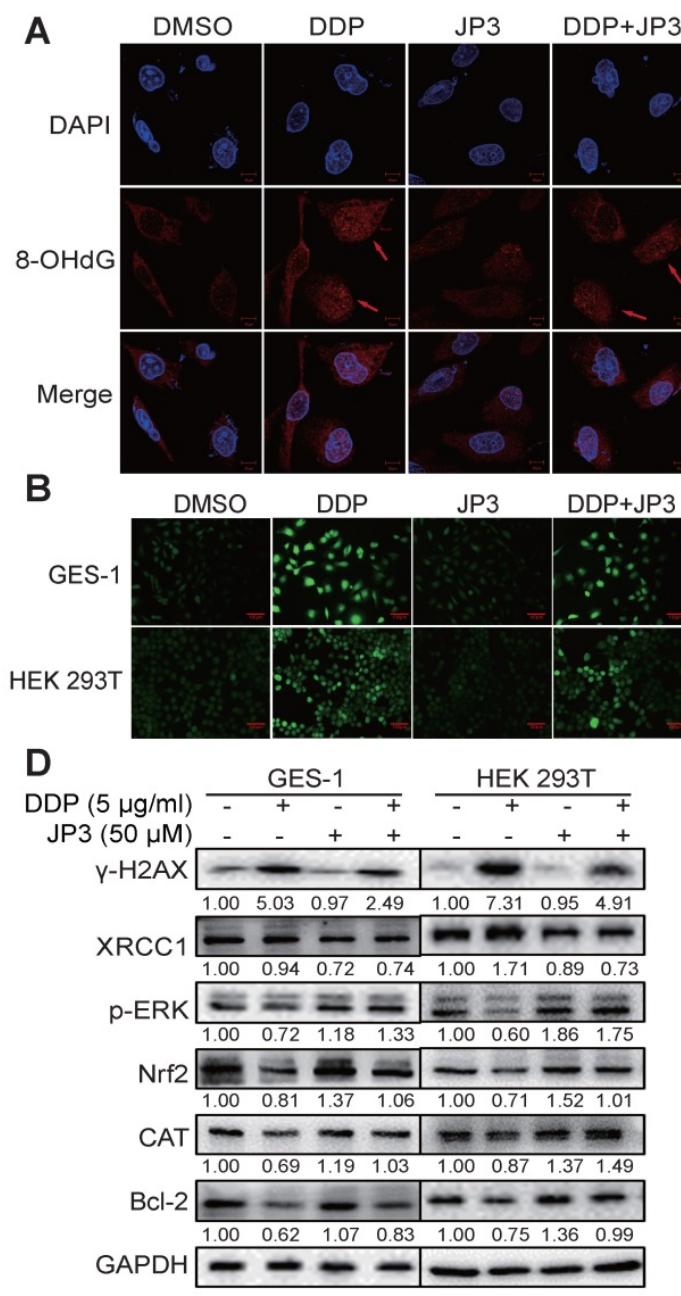

F

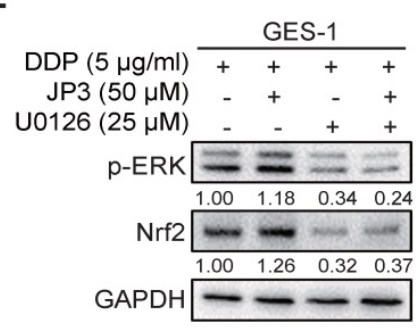

G
C

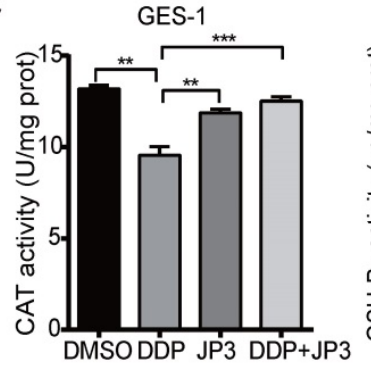
HEK 293T

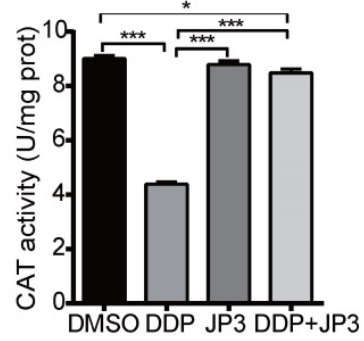

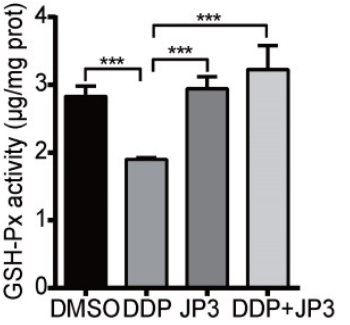

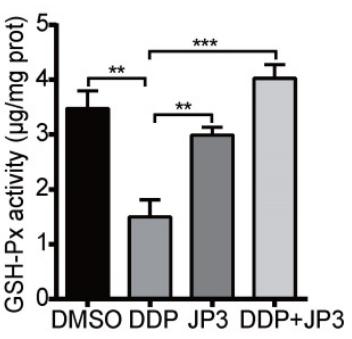

E
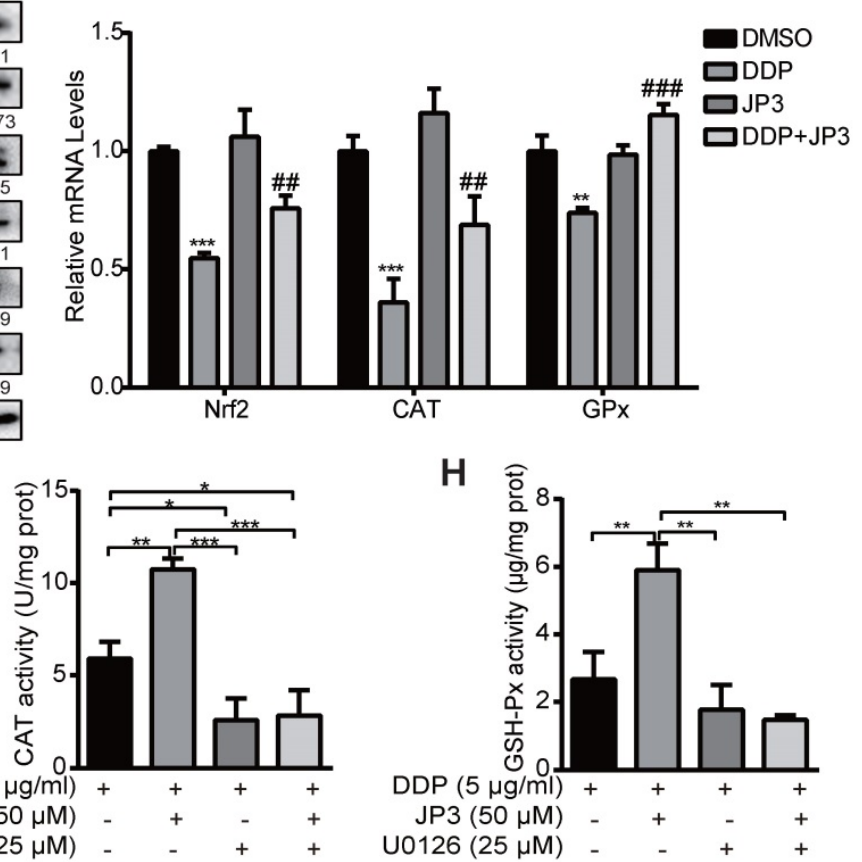

Figure 5. JP3 attenuates DDP-induced DNA damage in normal cells by reducing ROS. (A) GES- 1 cells were treated with DMSO, $5 \mu \mathrm{g} / \mathrm{ml} \mathrm{DDP}, 50 \mu \mathrm{M} J \mathrm{P} 3$ or DDP+JP3 for $24 \mathrm{~h}$; the images showed immunofluorescence staining of 8-OHdG (red) and DAPI (blue). Scale bars $=20 \mu \mathrm{m}$. (B) GES-1 and HEK $293 \mathrm{~T}$ cells were treated with DMSO, $5 \mu \mathrm{g} / \mathrm{ml}$ DDP, $50 \mu \mathrm{M}$ JP3 or DDP+JP3 for $24 \mathrm{~h}$; intracellular ROS levels were measured using a fluorescent dye (DCFH-DA). Scale bars = $100 \mu \mathrm{m}$. $(\mathrm{C}) \mathrm{The}$ content of intracellular activities of CAT and GSH-Px were measured by related commercial kits in both GES-1 and HEK293T cells under indicated treatments. (D) Protein levels of $\mathrm{Y}-\mathrm{H} 2 \mathrm{AX}, \mathrm{XRCC1}$, p-ERK, Nrf2, CAT and Bcl-2 were determined by western blot under indicated treatments; GAPDH was used as the loading control. (E) The mRNA levels of Nrf2, CAT and GPx in GES-1 cells were evaluated by qPCR assay, and the error bars indicate the means \pm S.D. $* P<0.05$, $* * P<0.01$, $* * * P<0.001$ compared with DMSO, and $\# P<0.05, \# P<0.01, \ldots+2>0.001$ compared with DDP treatment. (F) GES-1 cells were treated with $5 \mu \mathrm{g} / \mathrm{ml}$ DDP or $50 \mu M \mathrm{JP} 3$ alone or in combination, and with or without U $0126(25 \mu \mathrm{g} / \mathrm{ml})$ for $24 \mathrm{~h}$, then the protein levels of p-ERK, Nrf2 were determined by western blot; and activities of CAT and GSH-Px were determined by commercial kits (G-H). For all graphs, error bars indicate the means \pm S.D. $* P<0.05, * * P<0.01$, $* * * P<0.001$.

To elucidate the underlying mechanisms of JP3 in anti-DDP triggered oxidative stress, the intracellular antioxidant enzymes GSH-Px, CAT and SOD were also analyzed. As shown in Figure 5C, JP3 reversed DDP-induced declines in GSH-Px and CAT activities in GES-1 and HEK393T cells, however, JP3 has no significant role on DDP reduced SOD activity (Figure S2C).
We have previously identified that JWA rescues the accumulation of paraquat generated ROS via MEK/PI3K-Nrf2 signaling [19], here we determined if JP3 was also worked through this signaling. The result showed JP3 activated the phosphorylation of ERK and up-regulated both Nrf2 and CAT expressions and therefore reversed DDP mediated oxidative stresses in both GES-1 and HEK193T cells; 
in addition, JP3 increased $\mathrm{Bcl}-2$ expressions in the both cells (Figure 5D). As shown in Figure 5E, JP3 also transcriptional rescued DDP reduced expressions of Nrf2, GSH-Px and CAT in GES-1 cells. To confirm whether ERK associated signal pathway involved in anti-oxidant roles of JP3 in the normal cells, U0126 was used to block ERK signaling. As shown in Figure $5 \mathrm{~F}$, the pharmacological inhibition of ERK by U0126 abolished the effects of JP3 from DDP induced oxidative stress, and the elevated expression of Nrf2, and GSH-Px and CAT activities by JP3 were also blocked by U0126 (Figure 5G-H). These results suggest that JP3 through activating of ERK/Nrf2 linked anti-oxidant system protected normal cells from DDP induced oxidative stress and DNA damage; JP3 also enhanced anti-apoptosis potential in these cells.

\section{JP3 exerts bidirectional roles in BGC823/DDP xenografts model mice}

To verify whether JP3 has a similar bidirectional effect on DDP-treated GC in vivo, we established a BGC823/DDP GC cell xenograft mouse model. As shown in Figure 6A, compared with DDP treatment alone, the inhibition of tumor growth in DDP+JP3 group was enhanced obviously. The ratio of tumor/body weight was significantly lower in $\mathrm{DDP}+\mathrm{JP} 3$ group than that in DDP alone group (Figure 6B). Accordingly, the tumor inhibition rates were $7.4 \%, 43.0 \%$ and $75.8 \%$ in JP3, DDP and DDP+JP3 groups compared with the vehicle control group, respectively (Figure 6C-D). Moreover, histological examination revealed that the numbers of apoptotic cell was increased in tumor tissues obviously in $\mathrm{DDP}+\mathrm{JP} 3$ group compared to that in DDP group; and the inflammatory cell infiltration in kidney tissues were relieved in DDP+JP3 group compared with DDP group (Figure 6E). In addition, the significantly reduced GSH-Px and CAT activity in mouse kidney tissues treated with DDP alone was reversed in mouse treated with JP3 combined with DDP (Figure 6F). Finally, we determined expressions of related biomarkers in tumor tissues. As shown in Figure 6G, DDP treatment increased the protein levels of CK2a, XRCC1, p-XRCC1 and Bcl-2 were suppressed in both JP3 alone and JP3 plus DDP groups. In addition, the results of serum biochemical markers showed that although DDP treatment reduced LDH activities from $1036.3 \pm 164.59 \mathrm{U} / \mathrm{L}$ in untreated group to $747.5 \pm$ 131.37 U/L in DDP group, the enzymes of ALT (DDP vs control): $22.8 \pm 1.71$ vs $18.0 \pm 2.94$ and BUN (DDP vs control): $11.7 \pm 1.70$ vs $7.9 \pm 1.29$; CREA: $21.5 \pm 4.20$ vs $14.8 \pm 0.96)$ caused by DDP were also obviously. JP3 alone showed a reduction of LDH activity and an obvious protection role on kidney (CREA (JP3 vs control): $11.3 \pm 0.96$ vs $14.8 \pm 0.96)$; in JP3 plus DDP group, importantly, tumor burdens were further reduced compared to DDP alone group, however, liver and kidney functions were improved (Table S1). These data further confirmed a synergistic effects on cancer cells and a protecting effects on liver and kidney cells by JP3 when combined with DDP.

\section{Discussion}

DDP is a highly effective chemotherapeutic agent used in the treatment of solid tumors including GC, but its toxic side effects and secondary resistance are still two obvious challenges in the clinic [20]. In this study, we reported for the first time that although JP3 alone showed a weak suppression on DDP-resistant GC cells proliferation in vivo, it exerted bidirectional roles when used in combination with DDP. On the one hand, JP3 restored the sensitivity of the DDP resistant GC cells; on the other hand, JP3 reduced toxic side effects of DDP in model mice. The mechanism of action preliminarily elucidated in this study includes that JP3 weakened the DNA repair and anti-apoptosis ability of GC cells through CK2 $\alpha$-XRCC1 signaling pathway. JP3 also enhanced the antioxidant capacity of mouse, thus reduced the toxic and side effects of DDP (Figure 7).

Tumor cells generate DDP resistance by enhancing DNA damage repair capabilities [21]; XRCC1 plays an important role in the single strand break repair (SSB) and base excision repair (BER) [22, 23]. Expressions of XRCC1 and JWA protein in GC tissue is reported a prognostic and predictive role [24], and overexpression of JWA leads to the inhibition of CK2-p-XRCC1 - XRCC1 pathway [11]. Our current results revealed several novel connection and roles of JP3 in DDP-resistant GC. CK2 is highly expressed in a variety of cancers and is associated with tumor growth [25], it also synergistically works with other oncogenes [26, 27]; several CK2 inhibitors have been reported to be effective in reducing tumor resistance [28, 29]. In this study, CK2a expressions was down-regulated in mRNA transcription level by JP3 with or without DDP in time-dose dependent manner. It is worth pointing out that JP3 reduced activation of XRCC1 by competitively binding with CK2 $\alpha$ and thus CK2 $\alpha$ unable to activate XRCC1. Previously, we demonstrated that JWA accelerated the degradation of CK2a through the ubiquitination mediated mechanism, thus reduced the activation of XRCC1 [11]. Therefore, although JP3 and JWA protein could reduce XRCC1 activity through $\mathrm{CK} 2 \mathrm{a}$, their molecular mechanisms were completely different.

It is well known that DDP treatment induces a series of side effects including neurotoxicity, ototoxicity and gastro toxicity [30]. DDP is able to 
shift the redox balance in cells by conjugation leading to ROS over production [31], and therefore oxidative stress is the main mechanism underlying DDP-induced toxicity [32, 33]. JWA is involved in cellular responses to suppress environmental stress including oxidative stress [12]; JWA also protects neuronal cells from damage after exposure to the environmental toxicant $\mathrm{PQ}$ by inhibiting ROS production and increases glutathione content [19]. In the present study, GES-1 and HEK293T cells exposed to DDP underwent serious oxidative stress caused-DNA damage. JP3 combined with DDP treatment declined ROS by activating ERK and Nrf2, a key factor in oxidative stress response [34], and increasing expression levels of antioxidant enzymes, therefore protected cells from DDP induced toxicity.

Elevated copper levels is a well-documented metabolic difference between malignant cells and 'normal' cells [35, 36]. Rizvi A et al. have demonstrated that calcitriol, the metabolically active form of vitamin $\mathrm{D}$, interacted with copper and produced reactive oxygen species by Fenton-HaberWeiss like reactions which caused irreparable DNA damage in carcinoma cells and induced the consequent selective cell death of malignant cells but spare normal cells. The mechanism of calcitriolcopper interaction has been confirmed in malignancy like rabbit lymphocytes [37], human peripheral blood lymphocytes [38] and hepatocellular carcinoma rat modal [39]. It has been previously shown that cisplatin also interact with cellular copper, and this interact may in part, be responsible for its chemotherapeutic effects [40]. Whether the selective toxicity of JP3 is related to the difference of copper levels between normal and tumor cells requires further investigation. Our results showed that JP3 may prevent oxidative damage from DDP by scavenging and clearing reactive oxygen species. Whether JP3 affects enzymatic and non-enzymatic scavengers of reactive oxygen species in cancer cells or not needs more experiments.
A

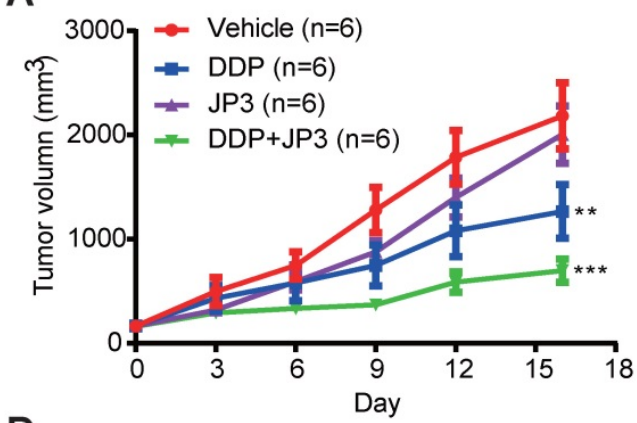

D

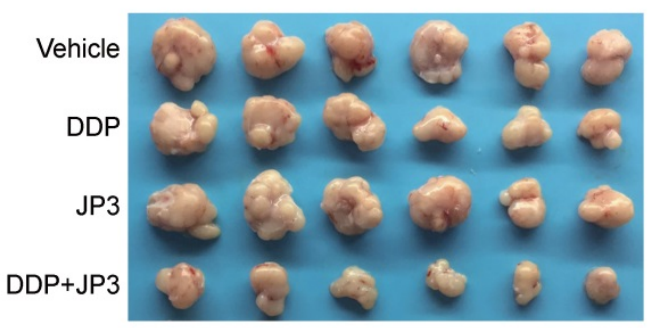

F Kidney
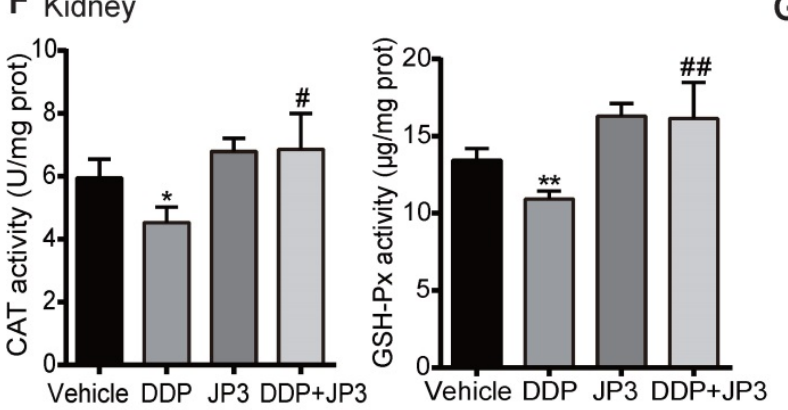

B

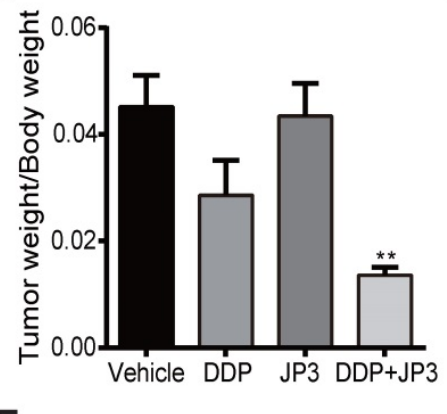

E

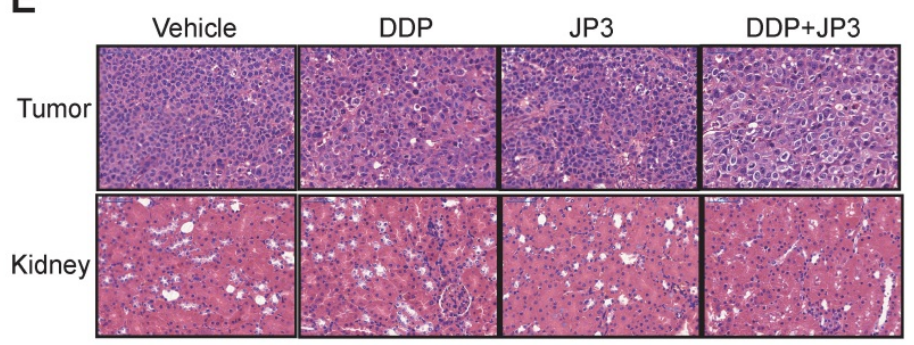

C

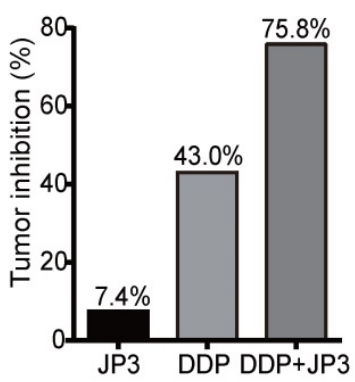

G

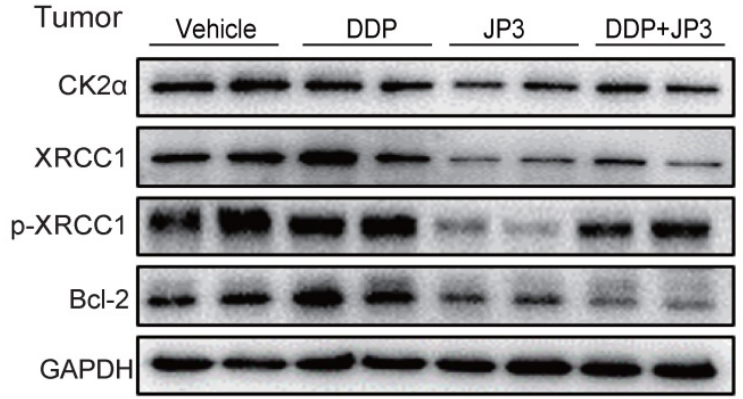

Figure 6. JP3 exerts bidirectional roles in BGC823/DDP xenografts model mice. (A) The tumor volumes of BGC823/DDP cell xenografts were measured every three days for all experimental mice, $n=6 ; * P<0.05$, $* * P<0.01$, $* * * P<0.001$ values were compared with vehicle group. (B) the ratio of tumor weight to body weight and the tumor inhibition rates $(C)$ were determined on day 16 after treatments; (D) the tumor mass images and representative H\&E staining images from tumors and kidney sections (original magnification: $200 \times$, scale bar: $50 \mu \mathrm{m}$ ) were shown in (E). (F) The activities of CAT and GSH-Px of kidney tissues from the four groups were determined by related commercial kits, and error bars indicate the means \pm S.D. $* P<0.05$, $* * P<0.01$, ***P $<0.001$ compared with vehicle, and \#P<0.05, \#P<0.01, \#\#P<0.001 compared with DDP treatment. (G) The representative protein levels of CK2 $\alpha, X R C C 1, p-X R C C 1, C K 2 \alpha$ and $\mathrm{Bcl}-2$ in tumor tissues were determined by western blot; GAPDH was used as the loading control. 

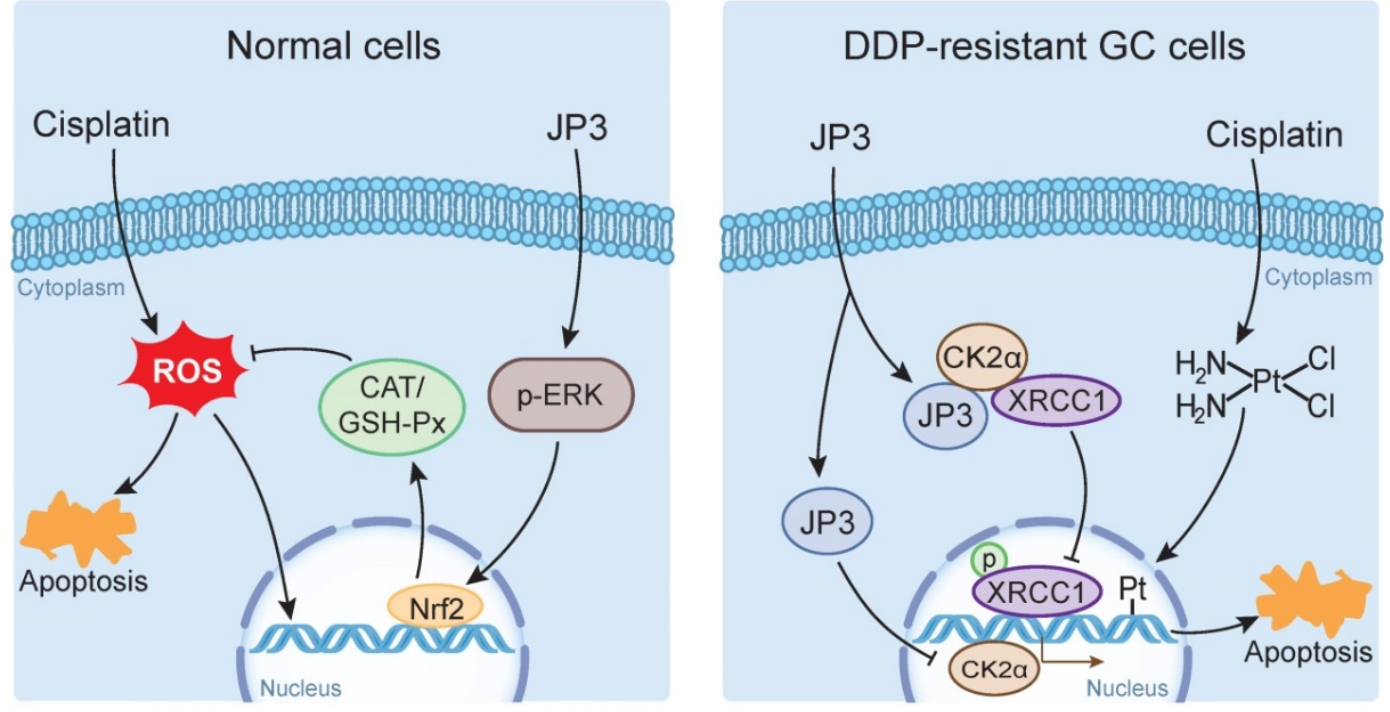

Figure 7. The bidirectional action mode and mechanism of JP3 combined with DDP in GC and normal cells

Elevated copper levels are a well-documented metabolic difference between malignant cells and 'normal' cells [35, 36]. Rizvi A et al. have demonstrated that calcitriol, the metabolically active form of vitamin D, interacted with copper and produced reactive oxygen species by Fenton-HaberWeiss like reactions which caused irreparable DNA damage in carcinoma cells and induced the consequent selective cell death of malignant cells but spare normal cells. The mechanism of calcitriolcopper interaction has been confirmed in malignancy like rabbit lymphocytes [37], human peripheral blood lymphocytes [38] and hepatocellular carcinoma rat modal [39]. It has been previously shown that cisplatin also interact with cellular copper, and this interact may in part, be responsible for its chemotherapeutic effects [40]. Whether the selective toxicity of JP3 is related to the difference of copper levels between normal and tumor cells requires further investigation. Our results showed that JP3 may prevent oxidative damage from DDP by scavenging and clearing reactive oxygen species. Whether JP3 affects enzymatic and non-enzymatic scavengers of reactive oxygen species in cancer cells or not needs more experiments.

The bidirectional effects of JP3 have also been demonstrated in vivo models. The efficacy of JP3 combined with DDP significantly decreased tumor growth compared to the monotherapy. Furthermore, the combined treatment also inhibited the expression of $\mathrm{CK} 2 \mathrm{a}, \mathrm{p}-\mathrm{XRCC} 1, \mathrm{XRCC} 1$ and the anti-apoptosis marker Bcl-2 [41]. Bcl-2 is known as an important anti-apoptotic gene, which can inhibit cell apoptosis and prolong cell survival. Many studies have confirmed that cisplatin resistance may relate with the high expression of Bcl-2 in tumor cells [41, 42]. In our study, JP3 treatment reduced the expression of Bcl-2 in DDP-resistant tissues and cells, but increased the expression of Bcl-2 in normal cells. Bcl-2 may also be a key point in the bidirectional effects of JP3, the specific mechanism needs further research. Our studies also showed that the inflammation and injury of kidney were relieved by enhancement of GSH-Px and CAT activities. The evidences in the present study indicate that JP3 in combination with DDP could be used as a potential treatment for reducing DDP resistance in GC cells and toxic side effects in mouse. Whether JP3 has a similar effect in combination with other chemotherapeutic drugs that produce oxidative stress or DNA damage needs further study. Since this study was conducted using cell culture models and cell lines based xenograft mouse models, the bidirectional effects of JP3 in combination with DDP need to be further validated in PDX models and other solid tumors. The potential value of JP3 as a single new anticancer drug also needs further evaluation.

Polypeptides, characterized by low molecular weight, high activity, low toxicity and easy modification, are widely concerned in anti-cancer experiments and clinical applications [43, 44]. JP3 is a pre-phosphorylated and MMP2-targeted 22 amino acids polypeptide, which simulates some biological functions of JWA protein. JP3 plays an important role in reducing cisplatin tolerance on GC cells and toxic side effects in mice. Our study may provide new insights for cisplatin in the treatment of drug-resistant gastric cancer.

\section{Abbreviations}

DDP: cisplatin; GC: gastric cancer; CK2: casein kinase 2; Nrf2: nuclear factor erythroid 2-related factor 2; XRCC1: X-ray repair cross complementing 
group1; SSB: single strand break; BER: base excision repair; ROS: reactive oxygen species; JWA: ADP-ribosylation-like factor 6 interacting protein 5; PARP1: poly(ADP-ribose) polymerase $1 ; \mathrm{\gamma}$-H2AX: phosphorylated histone H2AX; DAPI: $4^{\prime}$ 6-diamidino-2-phenylindole dihydrochloride; $\mathrm{CHX}$ : cycloheximide; CAT: catalase; SOD: superoxide dismutase; GPx: glutathione peroxidase; DCFH-DA: 2',7'-dichlorodihydrofluorescein diacetate; $\mathrm{IC}_{50}$ : half maximal inhibitory concentration.

\section{Supplementary Material}

Supplementary figures and tables. http://www.jcancer.org/v12p1894s1.pdf

\section{Acknowledgements}

This work was supported by the National Natural Science Foundation of China (Nos. 81520108027, 81521004, 81673219, 81973156 and 30930080).

\section{Author's contributions}

Conception and project design: Y. Zhang, J. Zhou Development of methodology: Y. Zhang, J. Chen, C, Shu, D. Chen, A. Li; Acquisition of data: Y. Zhang, J. Chen, C, Shu, D. Chen, A. Li; Analysis and interpretation of data: Y. Zhang, J. Chen, C. Shu, D. Chen, J. Zhou; Writing, review, and/or revision of the manuscript: Y. Zhang, J. Chen, C. Shu, J. Zhou; Administrative, technical, or material support: Y. Zhang, A. Li; Study supervision: J. Zhou.

\section{Competing Interests}

The authors have declared that no competing interest exists.

\section{References}

1. Bray F, Ferlay J, Soerjomataram I, Siegel RL, Torre LA, Jemal A. Global cancer statistics 2018: GLOBOCAN estimates of incidence and mortality worldwide for 36 cancers in 185 countries. CA Cancer J Clin. 2018; 68: 394-424.

2. Feng RM, Zong YN, Cao SM, Xu RH. Current cancer situation in China: good or bad news from the 2018 Global Cancer Statistics? Cancer Commun (Lond). 2019; 39: 22

3. Digklia A, Wagner AD. Advanced gastric cancer: Current treatment landscape and future perspectives. World J Gastroenterol. 2016; 22: 2403-14.

4. Rabik CA, Dolan ME. Molecular mechanisms of resistance and toxicity associated with platinating agents. Cancer Treat Rev. 2007; 33: 9-23.

5. Dugbartey GJ, Peppone LJ, de Graaf IA. An integrative view of cisplatin-induced renal and cardiac toxicities: Molecular mechanisms, current treatment challenges and potential protective measures. Toxicology. 2016; 371: $58-66$.

6. Galluzzi L, Senovilla L, Vitale I, Michels J, Martins I, Kepp O, et al. Molecular mechanisms of cisplatin resistance. Oncogene. 2012; 31: 1869-83.

7. Horton JK, Stefanick DF, Zhao ML, Janoshazi AK, Gassman NR, Seddon HJ, et al. XRCC1-mediated repair of strand breaks independent of PNKP binding. DNA Repair (Amst). 2017; 60: 52-63.

8. Sastry J, Kellie SJ. Severe neurotoxicity, ototoxicity and nephrotoxicity following high-dose cisplatin and amifostine. Pediatr Hematol Oncol. 2005; 22: 441-5.

9. Shahid F, Farooqui Z, Khan F. Cisplatin-induced gastrointestinal toxicity: An update on possible mechanisms and on available gastroprotective strategies. Eur J Pharmacol. 2018; 827: 49-57.

10. Huang $S$, Shen Q, Mao WG, Li AP, Ye J, Liu QZ, et al. JWA, a novel signaling molecule, involved in the induction of differentiation of human myeloid leukemia cells. Biochem Biophys Res Commun. 2006; 341: 440-50.
11. $\mathrm{X}_{\mathrm{u}} \mathrm{W}$, Chen $\mathrm{Q}$, Wang $\mathrm{Q}$, Sun $\mathrm{Y}$, Wang $\mathrm{S}, \mathrm{Li}$ A, et al. JWA reverses cisplatin resistance via the CK2-XRCC1 pathway in human gastric cancer cells. Cell Death Dis. 2014; 5: e1551.

12. Chen R, Qiu W, Liu Z, Cao X, Zhu T, Li A, et al. Identification of JWA as a novel functional gene responsive to environmental oxidative stress induced by benzo[a]pyrene and hydrogen peroxide. Free Radic Biol Med. 2007; 42: 1704-14.

13. Chen JJ, Ren YL, Shu CJ, Zhang Y, Chen MJ, Xu J, et al. JP3, an antiangiogenic peptide, inhibits growth and metastasis of gastric cancer through TRIM25/SP1/MMP2 axis. J Exp Clin Cancer Res. 2020; 39: 118.

14. Xu W, Wang S, Chen Q, Zhang Y, Ni P, Wu X, et al. TXNL1-XRCC1 pathway regulates cisplatin-induced cell death and contributes to resistance in human gastric cancer. Cell Death Dis. 2014; 5: e1055.

15. Zhou J, Ye J, Zhao X, Li A, Zhou J. JWA is required for arsenic trioxide induced apoptosis in HeLa and MCF-7 cells via reactive oxygen species and mitochondria linked signal pathway. Toxicol Appl Pharmacol. 2008; 230: 33-40.

16. Parsons JL, Dianova II, Finch D, Tait PS, Strom CE, Helleday T, et al. XRCC1 phosphorylation by CK2 is required for its stability and efficient DNA repair. DNA Repair (Amst). 2010; 9: 835-41.

17. Shahid F, Farooqui Z, Abidi S, Parwez I, Khan F, Oral administration of thymoquinone mitigates the effect of cisplatin on brush border membrane enzymes, energy metabolism and antioxidant system in rat intestine. Biomedicine \& Pharmacotherapy. 2017; 94: 1111-20.

18. Wang S, Gong Z, Chen R, Liu Y, Li A, Li G, et al. JWA regulates XRCC1 and functions as a novel base excision repair protein in oxidative-stress-induced DNA single-strand breaks. Nucleic Acids Res. 2009; 37: 1936-50.

19. Zhao X, Wang $\mathrm{R}$, Xiong $\mathrm{J}$, Yan D, Li A, Wang S, et al. JWA antagonizes paraquat-induced neurotoxicity via activation of Nrf2. Toxicol Lett. 2017; 277: $32-40$.

20. Amable L. Cisplatin resistance and opportunities for precision medicine. Pharmacol Res. 2016; 106: 27-36

21. Yan L, Lin M, Pan S, Assaraf YG, Wang ZW, Zhu X. Emerging roles of F-box proteins in cancer drug resistance. Drug Resist Updat. 2019; 49: 100673.

22. Lin Y, Raj J, Li J, Ha A, Hossain MA, Richardson C, et al. APE1 senses DNA single-strand breaks for repair and signaling. Nucleic Acids Res. 2020; 48: 1925-40.

23. Menoni $\mathrm{H}$, Wienholz $\mathrm{F}$, Theil AF, Janssens RC, Lans $\mathrm{H}$, Campalans A, et al. The transcription-coupled DNA repair-initiating protein CSB promotes XRCC1 recruitment to oxidative DNA damage. Nucleic Acids Res. 2018; 46: 7747-56

24. Wang S, Wu X, Chen Y, Zhang J, Ding J, Zhou Y, et al. Prognostic and predictive role of JWA and XRCC1 expressions in gastric cancer. Clin Cancer Res. 2012; 18: 2987-96.

25. Ruzzene $M$, Pinna LA. Addiction to protein kinase CK2. a common denominator of diverse cancer cells? Biochim Biophys Acta. 2010; 1804: 499-504.

26. Borgo C, Ruzzene M. Role of protein kinase CK2 in antitumor drug resistance. J Exp Clin Cancer Res. 2019; 38: 287.

27. Williams MD, Nguyen T, Carriere PP, Tilghman SL, Williams C. Protein Kinase CK2 Expression Predicts Relapse Survival in ERalpha Dependent Breast Cancer, and Modulates ERalpha Expression in Vitro. Int J Environ Res Public Health. 2015; 13: ijerph13010036.

28. Liu X, Chen J, Li W, Hang C, Dai Y. Inhibition of Casein Kinase II by CX-4945, But Not Yes-associated protein (YAP) by Verteporfin, Enhances the Antitumor Efficacy of Temozolomide in Glioblastoma. Transl Oncol. 2020; 13: 70-8.

29. Nitta RT, Bolin S, Luo E, Solow-Codero DE, Samghabadi P, Purzner T, et al. Casein kinase 2 inhibition sensitizes medulloblastoma to temozolomide. Oncogene. 2019; 38: 6867-79.

30. Hassan I, Chibber S, Naseem I. Vitamin B(2): a promising adjuvant in cisplatin based chemoradiotherapy by cellular redox management. Food Chem Toxicol. 2013; 59: 715-23.

31. Manohar S, Leung N. Cisplatin nephrotoxicity: a review of the literature. J Nephrol. 2018; 31: 15-25.

32. Antunes LM, Darin JD, Bianchi MD. Protective effects of vitamin c against cisplatin-induced nephrotoxicity and lipid peroxidation in adult rats: a dose-dependent study. Pharmacol Res. 2000; 41: 405-11.

33. Rosic G, Selakovic D, Joksimovic J, Srejovic I, Zivkovic V, Tatalovic N, et al. The effects of $\mathrm{N}$-acetylcysteine on cisplatin-induced changes of cardiodynamic parameters within coronary autoregulation range in isolated rat hearts. Toxicol Lett. 2016; 242: 34-46.

34. Wakabayashi N, Itoh K, Wakabayashi J, Motohashi H, Noda S, Takahashi S, et al. Keap1-null mutation leads to postnatal lethality due to constitutive Nrf2 activation. Nat Genet. 2003; 35: 238-45

35. Nasulewicz A, Mazur A, Opolski A. Role of copper in tumour angiogenesis--clinical implications. J Trace Elem Med Biol. 2004; 18: 1-8.

36. Gupte A, Mumper RJ. Elevated copper and oxidative stress in cancer cells as a target for cancer treatment. Cancer Treat Rev. 2009; 35: 32-46.

37. Rizvi A, Hasan SS, Naseem I. Selective cytotoxic action and DNA damage by calcitriol-Cu(II) interaction: putative mechanism of cancer prevention. PLoS One. 2013; 8: e76191.

38. Rizvi A, Chibber S, Naseem I. Cu(II)-vitamin D interaction leads to free radical-mediated cellular DNA damage: a novel putative mechanism for its selective cytotoxic action against malignant cells. Tumour Biol. 2015; 36: 1695-700. 
39. Rizvi A, Farhan M, Naseem I, Hadi SM. Calcitriol-copper interaction leads to non-enzymatic, reactive oxygen species mediated DNA breakage and modulation of cellular redox scavengers in hepatocellular carcinoma. Apoptosis. 2016; 21: 997-1007.

40. Xi Z, Guo W, Tian C, Wang F, Liu Y. Copper binding promotes the interaction of cisplatin with human copper chaperone Atox1. Chem Commun (Camb). 2013; 49: 11197-9.

41. Low SY, Tan BS, Choo HL, Tiong KH, Khoo AS, Leong CO. Suppression of BCL-2 synergizes cisplatin sensitivity in nasopharyngeal carcinoma cells. Cancer Lett. 2012; 314: 166-75.

42. Geng X, Liu L, Tsai KJ, Liu Z. Role of ZIP8 in regulation of cisplatin sensitivity through Bcl-2. Toxicol Appl Pharmacol. 2019; 362: 52-8.

43. Fosgerau K, Hoffmann T. Peptide therapeutics: current status and future directions. Drug Discovery Today. 2015; 20: 122-8.

44. Hu J, Cheng TAO, Zhang L, Sun B, Deng LEI, Xu H. Anti-tumor peptide AP25 decreases cyclin D1 expression and inhibits MGC-803 proliferation via phospho-extracellular signal-regulated kinase-, Src-, c-Jun N-terminal kinase-and phosphoinositide 3-kinase-associated pathways. Molecular Medicine Reports. 2015; 12: 4396-402. 